ARTICLE

\title{
A high capacity small molecule quinone cathode for rechargeable aqueous zinc-organic batteries
}

Zirui Lin (1) ${ }^{1}$, Hua-Yu Shi ${ }^{1}$, Lu Lin ${ }^{1}$, Xianpeng Yang ${ }^{1}$, Wanlong $\mathrm{Wu}^{1} \&$ Xiaoqi Sun (10 ${ }^{1 凶}$

Rechargeable aqueous zinc-organic batteries are promising energy storage systems with lowcost aqueous electrolyte and zinc metal anode. The electrochemical properties can be systematically adjusted with molecular design on organic cathode materials. Herein, we use a symmetric small molecule quinone cathode, tetraamino-p-benzoquinone (TABQ), with desirable functional groups to protonate and accomplish dominated proton insertion from weakly acidic zinc electrolyte. The hydrogen bonding network formed with carbonyl and amino groups on the TABQ molecules allows facile proton conduction through the Grotthusstype mechanism. It guarantees activation energies below $300 \mathrm{meV}$ for charge transfer and proton diffusion. The TABQ cathode delivers a high capacity of $303 \mathrm{mAh} \mathrm{g}^{-1}$ at $0.1 \mathrm{Ag}^{-1}$ in a zinc-organic battery. With the increase of current density to $5 \mathrm{Ag}^{-1}, 213 \mathrm{mAh} \mathrm{g}^{-1}$ capacity is still preserved with stable cycling for 1000 times. Our work proposes an effective approach towards high performance organic electrode materials.

\footnotetext{
${ }^{1}$ Department of Chemistry, Northeastern University, Shenyang, China. ${ }^{\varpi_{e}}$ mail: sunxiaoqi@mail.neu.edu.cn
} 
$\mathrm{R}$ echargeable lithium-ion batteries have been widely used in portable electronics and electric vehicles ${ }^{1-5}$. However, cost and potential safety issues restrict their application areas and scales ${ }^{6-10}$. Rechargeable aqueous batteries using aqueous electrolytes largely reduce those concerns and experience rapid development in the past few years. The zinc metal is a desirable anode for aqueous systems and delivers high theoretical specific capacity $\left(820 \mathrm{mAh} \mathrm{g}^{-1}\right)$, low redox potential (-0.76 V vs. S.H.E.) as well as good compatibility with water ${ }^{11-13}$. The research on suitable cathode materials to couple with the zinc anode mainly focuses on inorganic compounds such as metal oxides, Prussian blue salts and polyanion compounds ${ }^{14-17}$. Among them, manganese oxides and vanadium oxides deliver high capacities; ${ }^{18-21}$ however, problems such as active material dissolution are important challenges for their further applications ${ }^{14}$.

In comparison to inorganic materials, organic compounds are less explored as cathode materials for aqueous batteries ${ }^{22}$. Nevertheless, organic compounds are more flexible with molecular design, which allows the systematic adjustment of voltage, capacity, conductivity, redox kinetics and other properties for electrode materials ${ }^{23-25}$. For example, Koshika et al. attached the electrochemically active 2,2,6,6-tetramethylpiperidine-1-oxyl to the poly(vinyl ether) chain in order to avoid dissolution. The obtained electrode delivers $131 \mathrm{mAh} \mathrm{g}^{-1}$ capacity and excellent rate capability with the facile radical-involved redox process ${ }^{26}$. Our group showed that sulfo group can be introduced to the sixmembered carbon ring of polyaniline to function as an internal proton reservoir and retain a locally high acidic environment. The electrochemical activity of polyaniline is therefore maintained in the weakly acidic $\mathrm{Zn}$ electrolyte ${ }^{27}$.

Among organic materials, quinone compounds show good environmental friendliness. They widely exist in nature, and the redox process between quinone and hydroquinone is an important physiological progress in living organisms ${ }^{28}$. The excellent redox activity of quinones also makes them desirable cathode materials for aqueous zinc batteries. Zhao et al. reported a calix[4] quinone (C4Q) molecule, where each benzoquinone unit is connected by $\mathrm{C}-\mathrm{C}$ single bond to form a bowl-like structure. The cathode displays a high capacity of $335 \mathrm{mAh} \mathrm{g}^{-1}$ at $20 \mathrm{~mA} \mathrm{~g}^{-128}$. Nam and co-workers designed a medium molecular weight quinone compound, triangular macrocyclic phenanthrenequinone $(\mathrm{PQ}-\Delta)$, which allows the co-insertion of $\mathrm{Zn}^{2+}$ and water and delivers a specific capacity of $225 \mathrm{mAh} \mathrm{g}^{-1}$ at $30 \mathrm{~mA} \mathrm{~g}^{-1}{ }^{29}$. Guo et al. proposed a pyrene-4,5,9,10-tetraone (PTO) cathode, achieving $336 \mathrm{mAh} \mathrm{g}^{-1}$ capacity at $40 \mathrm{~mA} \mathrm{~g}^{-1} 30$.

$\mathrm{Zn}^{2+}$ de/intercalation is demonstrated for most quinone materials in zinc cells; however, the diffusion of multivalent cation could be sluggish ${ }^{31}$. On the other hand, the facile insertion of proton into cathode materials from weakly acidic zinc electrolytes is possible with the hydrolysis of $\mathrm{Zn}^{2+}$ to continuously generate proton ${ }^{31-33}$, and proton could take part in the redox process of quinones ${ }^{34-36}$. Besides, most of the previously studied quinone-based compounds are relatively complex in molecular structures and require multi-step synthesis procedures. In comparison, small molecule quinones provide the advantage of simple synthesis, corresponding to a reduced cost. However, many small quinone molecules, such as benzoquinone, are prone to sublimation. It leads to difficulties in electrode manufacturing, handling and storage. Another important concern of small molecules is their relatively high solubility, resulting in the shuttling of active material in electrolytes and capacity decay. Nevertheless, previous studies have shown that molecules with high symmetry possess low dipole moments and thus low solubilities in aqueous solutions ${ }^{37}$. Considering the above factors, we herein specifically apply a symmetric small molecule of tetraamino-p-benzoquinone (TABQ) as the cathode material for aqueous zinc-organic batteries. The four amino groups are available for protonation in weakly acidic zinc electrolytes, which would create a proton active environment and initiate proton insertion. At the same time, hydrogen bonds are formed among amino and carbonyl groups, so that the compound does not sublimate even at elevated temperatures. More importantly, the hydrogen bonding network allows facile proton conduction through the breaking and reforming of hydrogen bonds. The TABQ electrode thus functions with dominated proton de/ intercalation and experiences low activation energies for charge transfer and diffusion. Excellent electrochemical performance is achieved.

\section{Results}

The TABQ active material is soluble in N-Methyl-2-pyrrolidone (NMP), and it undergoes a dissolution-reprecipitation process during electrode manufacturing. Figure la shows the Fourier transform infrared (FT-IR) spectrum of the TABQ active material after drying out from NMP (no carbon and binder). The multiple absorption peaks between 3469 and $3262 \mathrm{~cm}^{-1}$ (purple part) belong to the $\mathrm{N}-\mathrm{H}$ stretching vibrations with different symmetry modes from the four amino groups in TABQ. Notably, a broad band shows up on the right side as highlighted in cyan. It suggests the redshift of part of the N-H stretching vibration peak, which is a typical behavior upon the formation of hydrogen bonds ${ }^{38,39}$. Another site available for forming hydrogen bonds with amino is oxygen on carbonyl in the case of TABQ. Correspondingly, the carbonyl stretching vibration at $1547 \mathrm{~cm}^{-1}$ in TABQ (pink part) experiences more than $100 \mathrm{~cm}^{-1}$ of redshift in comparison to $1661 \mathrm{~cm}^{-1}$ in benzoquinone (spectral database for organic compounds SDBS). Although the electron-donor property of amino also causes the redshift of carbonyl, such a significant shift results from additional effects, i.e., the hydrogen bonding ${ }^{37,40}$. Finally, the peaks in the range of $1700-1270 \mathrm{~cm}^{-1}$ shown in green belong to the vibrations of the six-membered carbon ring containing carbon-carbon single bonds, double bonds and their synergistic effects.

The above analysis on FT-IR demonstrates the formation of hydrogen bonds with the amino and carbonyl functional groups in TABQ molecules. It results in strong interactions between adjacent molecules and furthermore affects the physical and chemical properties. We studied the sublimation behavior of TABQ using the setup shown in Supplementary Fig. 1, and benzoquinone was applied as the comparing standard. When the benzoquinone powder was heated at $90^{\circ} \mathrm{C}$ for $3 \mathrm{~h}$ in the oven, no original powder is left and yellow crystals are condensed all over the flask which stayed at room temperature (Fig. 1b). It demonstrates the sublimation of benzoquinone. With TABQ, on the contrary, the original powder remains on the aluminum cover which stayed in the oven and the flask is completely clear (Fig. 1c). The same phenomenon is observed even with more elevated temperature of $150^{\circ} \mathrm{C}$ (Fig. 1d). In addition, thermogravimetric analysis (TGA) with constant temperature hold at 90 ${ }^{\circ} \mathrm{C}$ shows continuous weight loss of benzoquinone as a result of sublimation, whereas a stable weight evolution is obtained with TABQ (Fig. 1e). The above analysis confirms the introduction of hydrogen bonds effectively suppresses the sublimation of small molecule quinone. It would provide convenience for various electrode treatments.

The solubility of TABQ in $1 \mathrm{M} \mathrm{ZnSO}_{4}$, which is a conventional electrolyte for zinc batteries, was quantified by UV-vis analysis (Fig. 1f $)^{37}$. The saturated concentration was determined to be $1.7 \mathrm{mmol} \mathrm{L}^{-1}$. This low solubility would ensure stable cycling in aqueous zinc cells. The relationship between molecular structure and solubility is further studied by comparing with related 

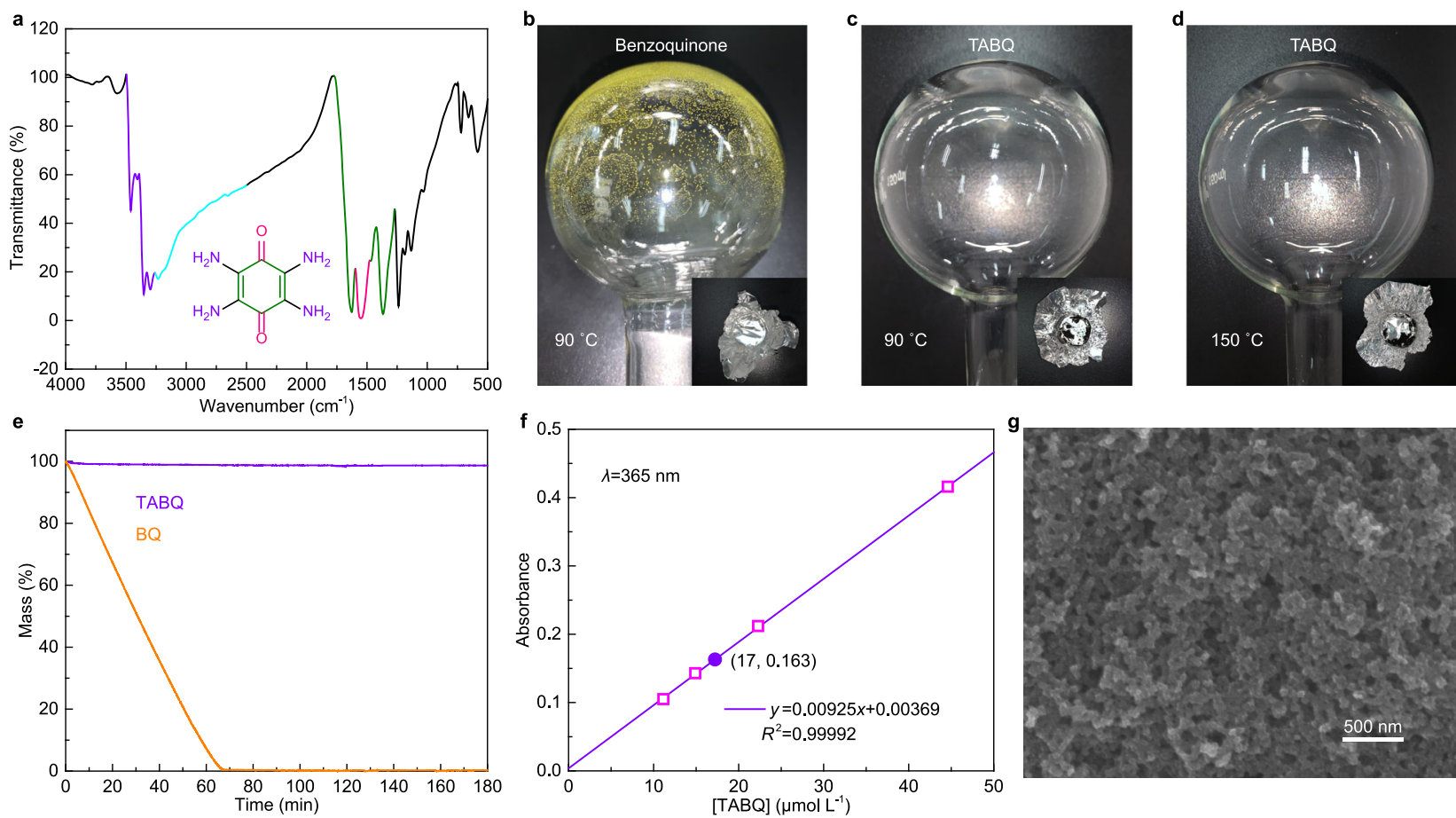

Fig. 1 Characterizations of TABQ. a FT-IR spectrum of $T A B Q$. b, c, d Sublimation test of benzoquinone and $T A B Q$ at $90{ }^{\circ} \mathrm{C}$ and $150{ }^{\circ} \mathrm{C}$. e $T G A$ curves of benzoquinone and $\mathrm{TABQ}$ with constant temperature hold at $90^{\circ} \mathrm{C}$. $\mathbf{f} U \mathrm{~V}$-vis calibration curve of $\mathrm{TABQ}$ in $1 \mathrm{M}_{\mathrm{ZnSO}}$ together with the calculation of the 100 times diluted saturated solution. $\mathbf{g}$ SEM image of the TABQ electrode.

compounds of tetrahydroxy-p-benzoquinone (THBQ), tetrachloroo-benzoquinone (o-TCBQ) and tetrachloro-p-benzoquinone ( $\mathrm{p}$ TCBQ). UV-vis analysis results in the solubilities of $3.4 \mathrm{mmol} \mathrm{L}^{-1}$ and $2.3 \mathrm{mmol} \mathrm{L}^{-1}$ for THBQ and o-TCBQ, respectively (Supplementary Fig. 2), and very trace dissolution of p-TCBQ is noticed. Literature also demonstrated the insoluble nature of $\mathrm{p}^{-T C B Q}{ }^{41}$. Comparing among the $\mathrm{p}$-quinones, the higher solubilities of TABQ and THBQ than p-TCBQ should be attributed to the more hydrophilic amino or hydroxyl functional groups. Nevertheless, the solubilities are still quite low thanks to their highly symmetric molecular structures and thus low dipole moments ${ }^{37}$. For example, the $\mathrm{p}$-TCBQ molecule is more symmetric than o-TCBQ and presents lower solubility.

Figure $1 \mathrm{~g}$ shows the scanning electron microscopy (SEM) image of the TABQ electrode. The TABQ active material forms as thin layer and is homogeneously distributed over Ketjen Black $(\mathrm{KB})$ spherical particles, resulting in a conductive network with porous structure. The effective interaction between TABQ and carbon would ensure good electrical conductivity in electrodes. Comparing with the high crystallinity of as-prepared TABQ, the $\mathrm{X}$-ray diffraction (XRD) pattern of TABQ electrode shows broad peaks due to short coherent lengths in the thin layer (Supplementary Fig. 3). The diffractions can be attributed to the parallel stacking of six-membered carbon rings in the quinone structure $^{41}$.

The electrochemical performance of the TABQ cathode was tested in zinc-organic cells with zinc foil anode and $1 \mathrm{M} \mathrm{ZnSO}_{4}$ aqueous electrolyte. The two most commonly used salts in zinc electrolytes are $\mathrm{ZnSO}_{4}$ and $\mathrm{Zn}\left(\mathrm{CF}_{3} \mathrm{SO}_{3}\right)_{2}$. They provide similar performance for TABQ (discussed later), and $\mathrm{ZnSO}_{4}$ was selected considering the lower price. Figure $2 \mathrm{a}, \mathrm{b}$ shows the voltage profiles and capacity evolution at various current densities. The cathode delivers $303 \mathrm{mAh} \mathrm{g}^{-1}$ capacity at the current density of $0.1 \mathrm{~A} \mathrm{~g}^{-1}$. The Ketjen Black (KB) conductive agent provides less than 25 $\mathrm{mAh}^{-1}$ capacity without any redox peaks in the differential capacity curve (Supplementary Fig. 4), demonstrating the capacity is majorly contributed by the TABQ active material. With the increase of current density to $5 \mathrm{Ag}^{-1}$, the TABQ cathode still maintains $213 \mathrm{mAh} \mathrm{g}^{-1}$ capacity. It suggests the excellent electrochemical activity of both carbonyl groups on TABQ. Figure $2 c$ shows the differential capacity curve at $0.1 \mathrm{~A} \mathrm{~g}^{-1}$. Two pairs of redox peaks are observed at $0.90 \mathrm{~V} / 1.02 \mathrm{~V}$ and $0.77 \mathrm{~V} / 0.83 \mathrm{~V}$, respectively. The small overpotential demonstrates the good cation de/insertion kinetics into TABQ. The electrochemical performance of TABQ is compared with previously reported quinone cathode materials in zinc-organic batteries, including $\mathrm{C}_{4} \mathrm{Q}^{28}, \mathrm{PQ}-\Delta^{29}, \mathrm{PTO}^{30}, \mathrm{TCBQ}^{41}$, $\mathrm{HqTp}^{42}$, poly(benzoquinonyl sulfide) (PBQS) ${ }^{43}$ and dibenzo[b,i] thianthrene-5,7,12,14-tetraone (DTT) ${ }^{44}$. As shown in Fig. 2d, TABQ delivers the highest capacity at $0.1 \mathrm{~A} \mathrm{~g}^{-1}$ and presents the best rate capability. Figure 2e and Supplementary Fig. 5 show the long-term cycling behavior. At the current density of $5 \mathrm{Ag}^{-1}$, the TABQ cathode exhibits stable capacity retention for over 1000 cycles after slight capacity decay during the first few cycles. The coulombic efficiencies are close to $100 \%$. FT-IR and SEM analysis on the electrode after 1000 cycles verifies the excellent compositional and morphological stabilities of TABQ (Supplementary Fig. 6), which ensures the superior cycling performance.

The stability of TABQ in zinc cells was further confirmed by a rest test, where the cell was cycled and rested for $10 \mathrm{~h}$ after discharged to half capacity, fully discharged, charged to half capacity and fully charged, respectively (Supplementary Fig. 7a). It corresponds to a total of $40 \mathrm{~h}$ rest period, allowing any possible dissolution to take place. Supplementary Fig. $7 \mathrm{~b}-\mathrm{d}$ shows the capacity retention, charge-discharge and differential capacity curves during the test. They are well preserved before, during and after the rest cycle, verifying the excellent stability of TABQ at various states. Another $\mathrm{Zn}$-TABQ cell was assembled with the electrolyte of $1 \mathrm{M} \mathrm{ZnSO}_{4}$ containing saturated TABQ and cycled at $0.1 \mathrm{Ag}^{-1}$. The anode does not show any nitrogen signal by $\mathrm{X}$ ray photoelectron spectroscopy (XPS) after 10 cycles (Supplementary Fig. 8). It demonstrates that the small amount of TABQ dissolved in electrolyte does not react with $\mathrm{Zn}$ and there is no 

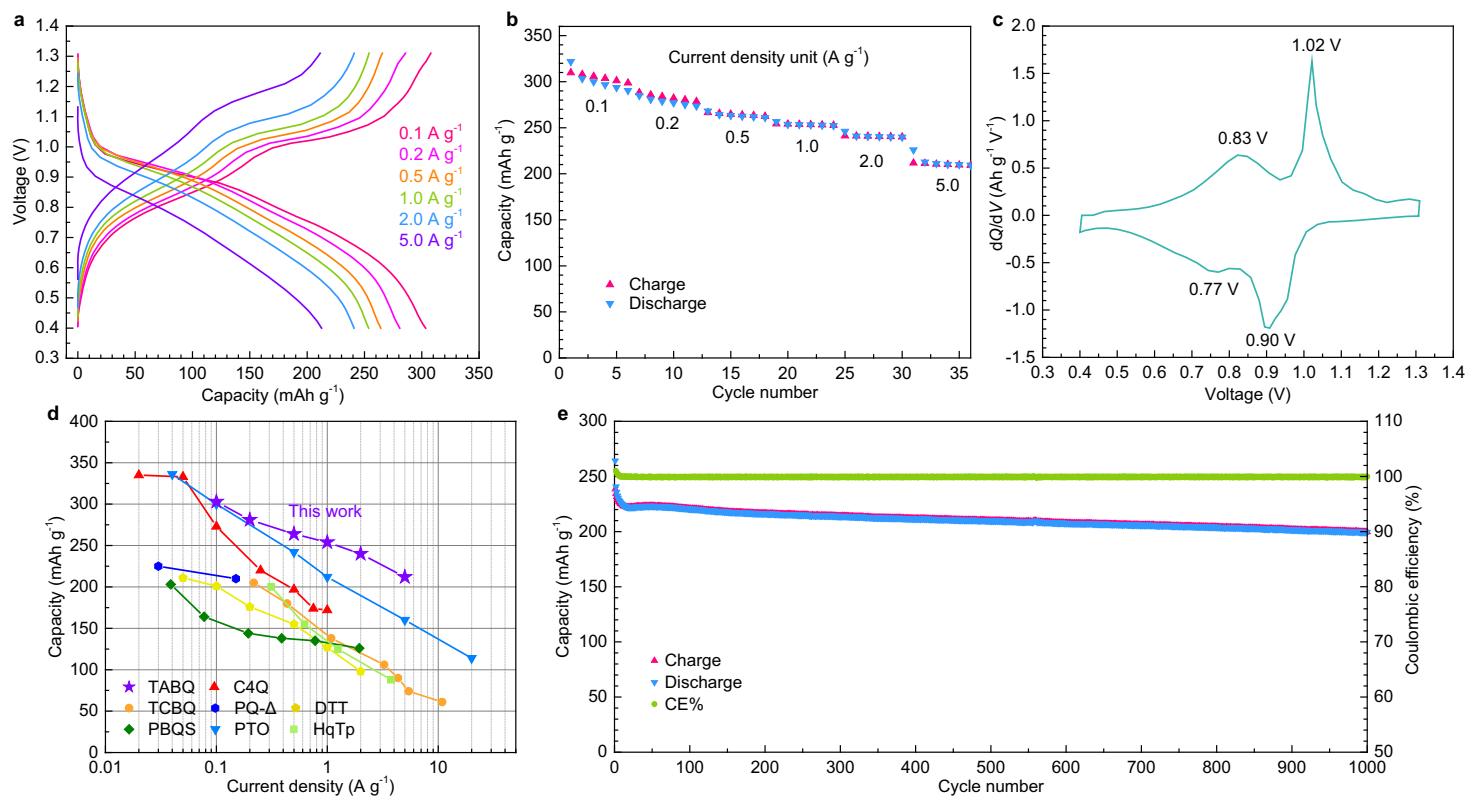

Fig. 2 Electrochemical performance of the TABQ cathode in aqueous zinc-organic batteries. a Charge/discharge curves and $\mathbf{b}$ capacity evolution under various current densities. c Differential capacity curve at $0.1 \mathrm{~A} \mathrm{~g}^{-1}$. d Comparison with previously reported quinone materials. e Capacity and coulombic efficiency evolution at $5 \mathrm{~A} \mathrm{~g}^{-1}$ for 1000 cycles.
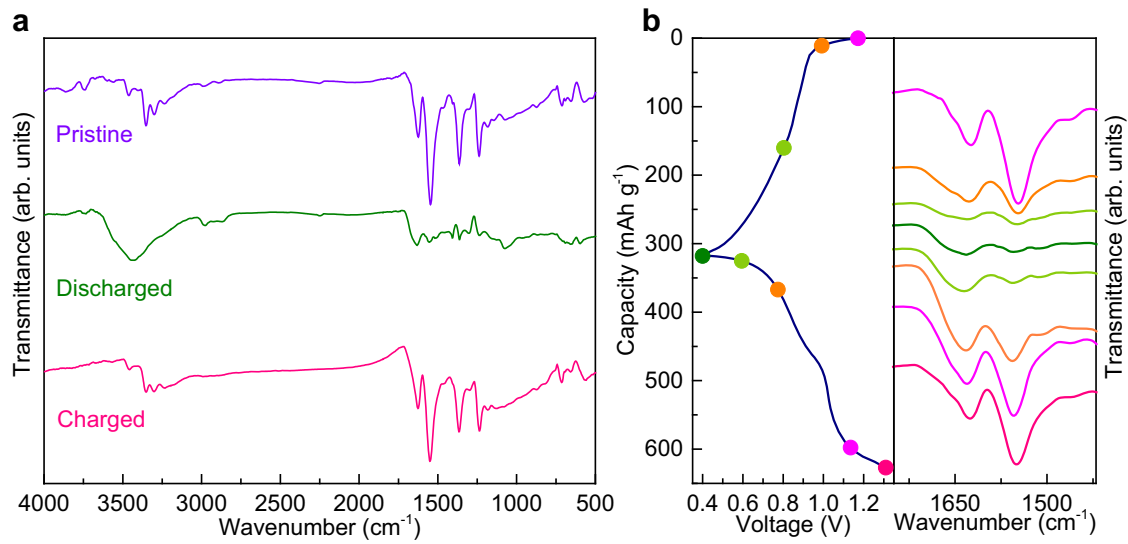

Fig. 3 Ex-situ FT-IR characterizations of TABQ. a FT-IR spectra of the TABQ electrode at the pristine, discharged and charged states. b Detailed FT-IR peak evolution along discharge and charge.

shuttling of TABQ. Overall, the excellent cycling stability of $\mathrm{Zn}$ TABQ cells is confirmed.

The evolution of TABQ during charge and discharge was studied by ex-situ FT-IR (Fig. 3a). Upon discharge, the carbonyl peak at $1547 \mathrm{~cm}^{-1}$ disappears, and the stretching vibration of carbon-carbon double bond at $1627 \mathrm{~cm}^{-1}$ shows much reduced intensity. The disappearance of carbonyl vibration is a result of its reduction during the discharge process. More specifically, $-\mathrm{C}=\mathrm{O}$ accepts electron and transforms into $-\mathrm{C}^{-} \mathrm{O}^{-}$anion. Simultaneously, cations insert to compensate the negative charge ${ }^{34-36,45}$. This reduction process also converts the conjugated quinone ring to the $\pi$-conjugated benzene ring. Since the quinone structure exhibits symmetric double bonds in the six-membered carbon ring while the benzene ring does not, the former provides larger dipole moment change of anti-symmetric stretching vibration and therefore shows stronger IR absorption. Upon charge, the FT-IR spectrum resembles the one for the pristine electrode, confirming the reversible redox of carbonyl group and transition between conjugated structures. Figure $3 \mathrm{~b}$ shows the more detailed peak evolution with measurements taken at various states along discharge and charge.
In addition, a periodic peak shape change is observed in the range of $3000-4000 \mathrm{~cm}^{-1}$ (Fig. 3a). At the end of discharge, a broad band appears and overlaps the original peaks of amino groups. The band disappears and the amino vibrations are revealed upon charge. The broad band is the characteristic absorption of water. The electrode was further studied to track its origin. Figure 4a shows the XRD of the electrode at different states. The change on the TABQ diffractions results from the transformation between conjugated quinone rings and $\pi$ conjugated benzene rings which exhibit different stacking distances ${ }^{41}$. The narrower peaks in the charged electrode suggest an enhanced long-range ordering. More significantly, the diffraction peak of $\mathrm{Zn}_{4} \mathrm{SO}_{4}(\mathrm{OH})_{6} \cdot 4 \mathrm{H}_{2} \mathrm{O}$ shows up in the discharged electrode, and it disappears upon charge. The same change is also noted in an in-situ XRD cell (Supplementary Fig. 9), suggesting the reversible formation of $\mathrm{Zn}_{4} \mathrm{SO}_{4}(\mathrm{OH})_{6} \cdot 4 \mathrm{H}_{2} \mathrm{O}$ at the cathode during the electrochemical processes. The SEM image of the electrode shows the reversible appearance and disappearance of platelets over the pristine nano-particles upon discharge and charge (Fig. 4b), which is the typical morphology of zinc sulfate 


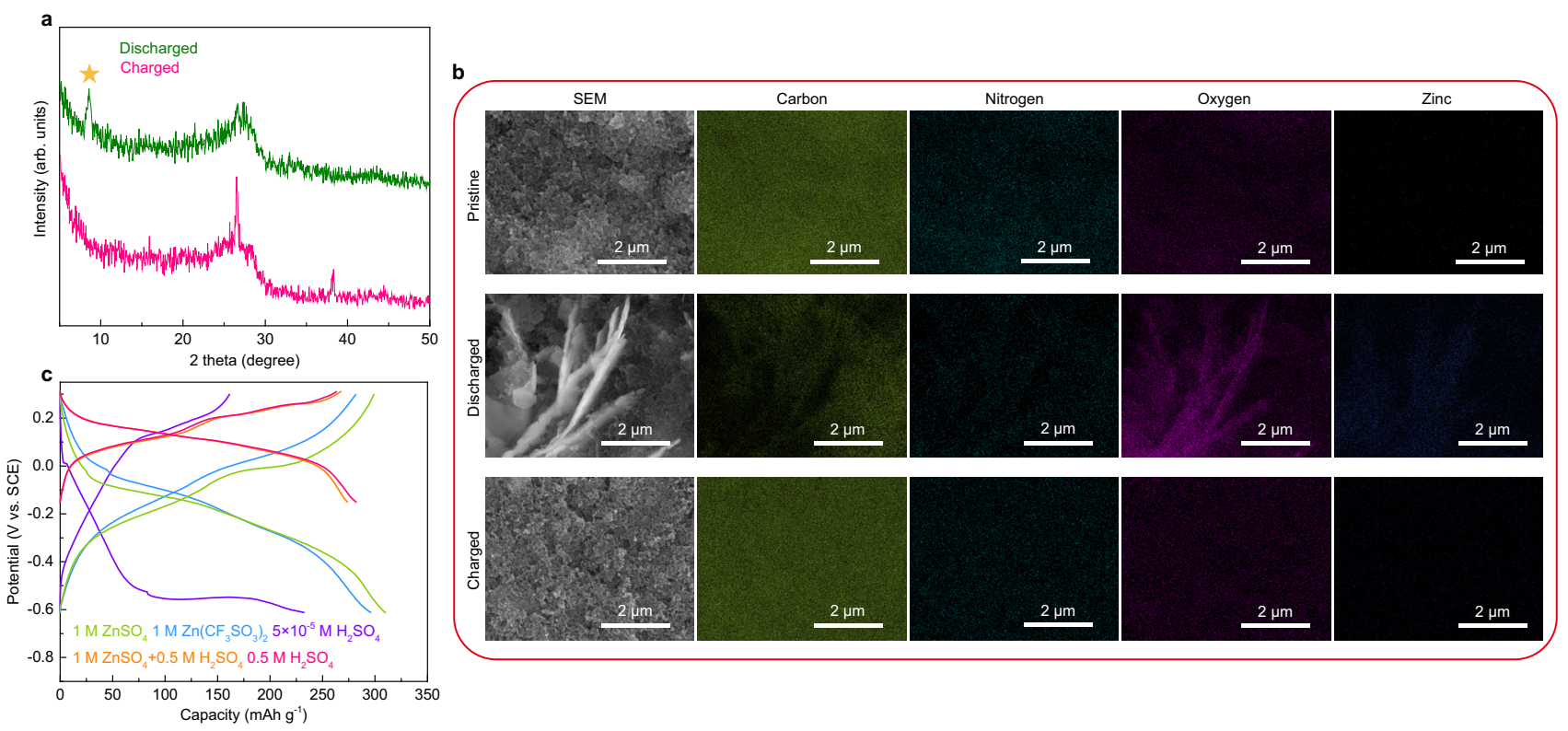

Fig. 4 Proof of proton insertion in TABQ. a XRD of TABQ at the end of discharge and charge (the asterisk shows the characteristic diffraction peak of $\left.\mathrm{Zn}_{4} \mathrm{SO}_{4}(\mathrm{OH})_{6} \cdot 4 \mathrm{H}_{2} \mathrm{O}\right)$. b SEM images and EDS mappings of TABQ at different states. $\mathbf{c}$ Charge/discharge curves of TABQ in three electrode cells with SCE reference electrode and different electrolytes.

hydroxide ${ }^{19}$. Energy dispersive X-ray spectroscopy (EDS) analysis gives a $\mathrm{Zn}: \mathrm{S}$ ratio around 4 in the discharged electrode (Supplementary Fig. 10), in agreement with the $\mathrm{Zn}_{4} \mathrm{SO}_{4}(\mathrm{OH})_{6} \cdot 4 \mathrm{H}_{2} \mathrm{O}$ composition. The elemental mappings further suggest that the platelets formed at the discharged state are $\mathrm{Zn}$-rich. The above results demonstrate that $\mathrm{Zn}_{4} \mathrm{SO}_{4}(\mathrm{OH})_{6} \cdot 4 \mathrm{H}_{2} \mathrm{O}$ is formed upon discharge and its crystal water gives rise to the broad band in FTIR. Since $\mathrm{Zn}_{4} \mathrm{SO}_{4}(\mathrm{OH})_{6} \cdot 4 \mathrm{H}_{2} \mathrm{O}$ precipitation takes place in solutions with $\mathrm{pH}$ above $5.5^{46,47}$, its formation in $\mathrm{ZnSO}_{4}$ electrolyte is due to proton insertion into cathode which leaves $\mathrm{OH}^{-}$behind and causes local $\mathrm{pH}$ increase ${ }^{19,48,49}$. It suggests reversible proton de/insertion into TABQ during charge/discharge. Despite the insulating nature of $\mathrm{Zn}_{4} \mathrm{SO}_{4}(\mathrm{OH})_{6} \cdot 4 \mathrm{H}_{2} \mathrm{O}$, it forms as individual platelets rather than uniform film over active material. Therefore, the electrical conductivity of the electrode is not hindered as confirmed by electrochemical impedance spectroscopy (EIS, Supplementary Fig. 11). Its reversible formation also functions as a $\mathrm{pH}$ buffer for the system.

Since $\mathrm{Zn}^{2+}$ would also function as the charge carrier in the $\mathrm{ZnSO}_{4}$ electrolyte, the amount of proton vs. $\mathrm{Zn}^{2+}$ storage in TABQ was quantified by inductively coupled plasma optical emission spectroscopy (ICP-OES) and ion chromatography (IC). They result in the $\mathrm{Zn}$ and sulfate weight percentages of $17.34 \%$ and $5.76 \%$ in the discharged electrode, respectively, and the inserted proton is calculated to be 13.5 times of $\mathrm{Zn}^{2+}$ (please find detailed calculations in Supplementary Discussions). It suggests the domination of proton storage in TABQ during the redox processes.

The dominated proton insertion in TABQ was further verified by the investigation of $\mathrm{pH}$ influence on cathode behaviors. The TABQ electrode was tested in three electrode cells with saturated calomel electrode (SCE) as the reference and a series of electrolytes: group A of $\mathrm{pH} 4$ solutions, including $1 \mathrm{M} \mathrm{ZnSO}_{4}, 1 \mathrm{M} \mathrm{Zn}$ $\left(\mathrm{CF}_{3} \mathrm{SO}_{3}\right)_{2}$ and $5 \times 10^{-5} \mathrm{M} \mathrm{H}_{2} \mathrm{SO}_{4}$; group $\mathrm{B}$ of $\mathrm{pH} 0$ solutions, including $0.5 \mathrm{M} \mathrm{H}_{2} \mathrm{SO}_{4}$ and $1 \mathrm{M} \mathrm{ZnSO}_{4}+0.5 \mathrm{M} \mathrm{H}_{2} \mathrm{SO}_{4}$. The average charge/discharge potentials of TABQ in the $\mathrm{pH} \sim 0$ electrolytes (group B) are around $0.13 \mathrm{~V}$ vs. SCE, while the ones with the $\mathrm{pH} \sim 4$ electrolytes (group A) are around $-0.12 \mathrm{~V}$ vs. SCE (Fig. 4c). Such $\mathrm{pH}$ dependent redox potential of TABQ demonstrates proton involved reaction, and the potential difference obeys the Nernst equation (please find detailed calculations in Supplementary Discussions). The electrode presents high overpotential in the $5 \times 10^{-5} \mathrm{M} \mathrm{H}_{2} \mathrm{SO}_{4}$ electrolyte due to the low ion activity, but the average potential is close to the other $\mathrm{pH} 4$ electrolytes. In $1 \mathrm{M} \mathrm{ZnSO}_{4}$ and $\mathrm{Zn}\left(\mathrm{CF}_{3} \mathrm{SO}_{3}\right)_{2}$, on the other hand, continuous $\mathrm{Zn}^{2+}$ hydrolysis provides enough proton for cathode reactions. The results confirm that proton is the dominated active cation associated with the redox of TABQ.

Importantly, the performance of zinc anode is not influenced by the proton de/insertion at the cathode, thanks to the reversible formation of $\mathrm{Zn}_{4}(\mathrm{OH})_{6} \mathrm{SO} \cdot 4 \mathrm{H}_{2} \mathrm{O}$ as $\mathrm{pH}$ buffer. Supplementary Fig. 12 shows the potential curves of zinc electrode in a threeelectrode cell with SCE reference and TABQ counter electrode. The flat and stable curves at different cycles demonstrate the excellent stability of Zn plating/stripping reaction in the system. It allows the coupling of zinc metal anode with a proton inserted cathode in the zinc-organic battery.

The unique proton insertion manner in TABQ should be attributed to its amino groups, which are available to protonate in the weakly acidic electrolyte of $\mathrm{ZnSO}_{4}$. It allows continuous proton exchange with electrolyte to create a proton active environment, which initiates proton insertion and helps with desolvation. The protonation of TABQ was confirmed by UV-vis analysis. In the non-protonated TABQ, the lone pair electrons on the amino groups would form extended conjugation with the $\pi$ electrons on quinone rings ${ }^{50,51}$. Such interaction is disturbed upon the protonation of amino groups. Therefore, the K-band in protonated TABQ possesses similar energy with benzoquinon ${ }^{52}$ and is blue-shifted in comparison to non-protonated TABQ due to the absence of extended conjugation. Figure $5 \mathrm{a}$ shows the UVvis spectra of TABQ in water and $1 \mathrm{M} \mathrm{ZnSO}_{4}$. A clear blueshift of K-band from $268 \mathrm{~nm}$ in water to $201 \mathrm{~nm}$ in $1 \mathrm{M} \mathrm{ZnSO}_{4}$ is noted, confirming the protonation in the latter.

The effect of protonation on proton insertion is further extended to the compounds of 2,5-diamino-1,4-benzoquinone $(\mathrm{DABQ})^{37}$, THBQ and 2,5-dihydroxy-1,4-benzenediacetate $(D O B D A)^{53}$. Supplementary Fig. 13a shows their chargedischarge curves, and the discharged electrodes were 

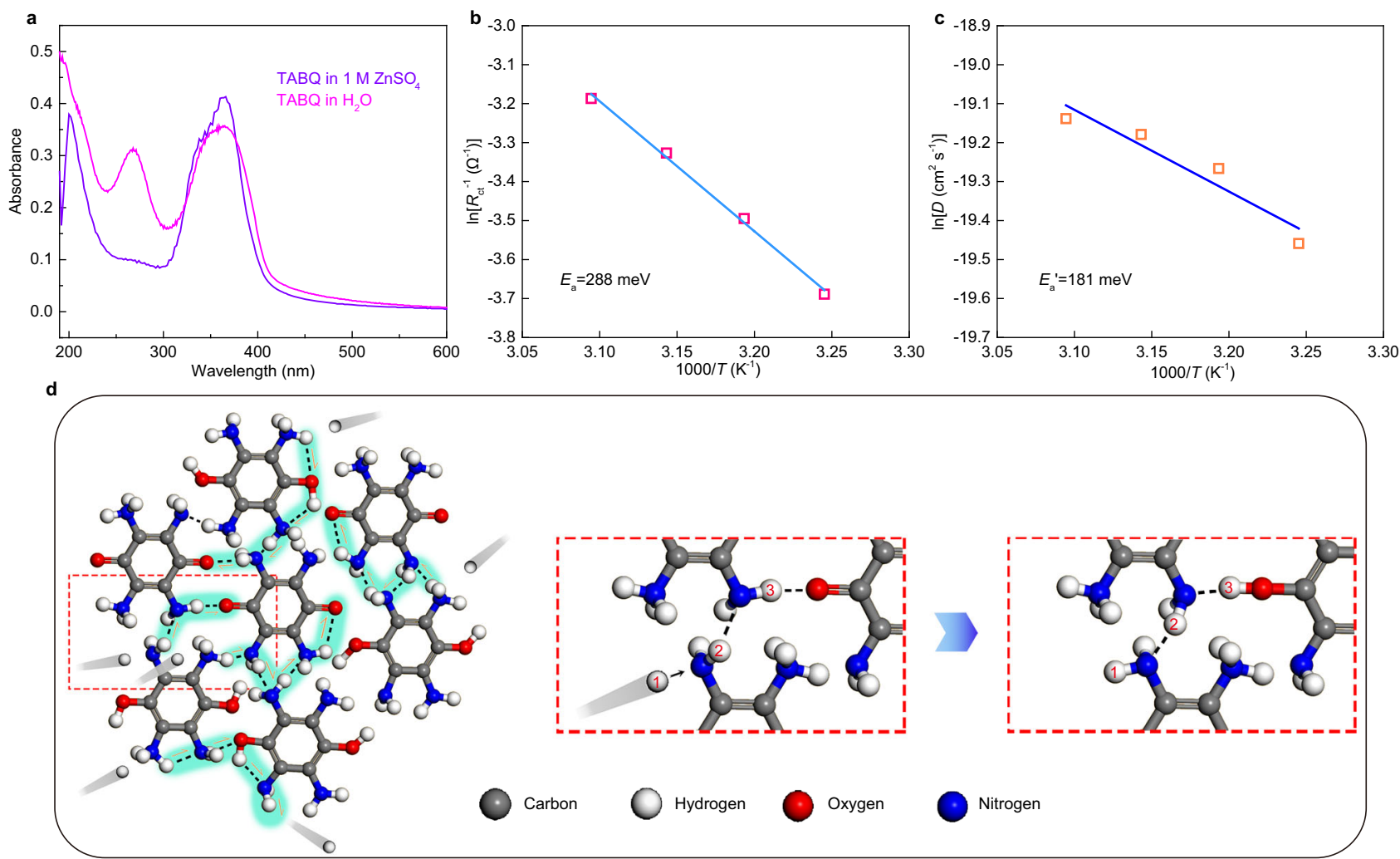

Carbon $\quad$ Hydrogen

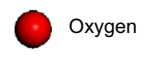

Nitrogen

Fig. 5 Proton insertion behavior into TABQ. a UV-vis absorption spectra of TABQ in $1 \mathrm{M} \mathrm{ZnSO}$ and water. The Arrhenius plots of $\mathbf{b} \ln \left(R_{\mathrm{ct}}\right)$ vs. $1000 / T$ and $\mathbf{c} \ln (D)$ vs. $1000 / T$ for $T A B Q$. d Schematic illustration for the proton conduction manner in the hydrogen bonding network in TABQ.

characterized by XRD (Supplementary Fig. 13b). The $\mathrm{Zn}_{4} \mathrm{SO}_{4}(\mathrm{OH})_{6} \cdot 4 \mathrm{H}_{2} \mathrm{O}$ diffraction peak is shown in the discharged $\mathrm{DABQ}$ and DOBDA, which indicates proton insertion. It is attributed to the active protonation/deprotonation of amino or carboxyl groups. On the other hand, the proton activity on hydroxyl groups is low in $1 \mathrm{M} \mathrm{ZnSO}_{4}^{54}$, and the protonation of carbonyl groups on quinones is negligible ${ }^{55-57}$. Proton insertion is thus less favored in THBQ. Previously reported quinone materials undergoing $\mathrm{Zn}$ storage, such as TCBQ, PTO and $\mathrm{C} 4 \mathrm{Q}^{28,30,41}$, would not protonate in the weakly acidic zinc electrolytes, either.

The charge transfer behavior in TABQ was studied by EIS with measurements taken at various temperatures. A typical equivalent circuit was applied to calculate charge transfer resistance $\left(R_{\mathrm{ct}}\right)$ from the Nyquist plots (Supplementary Fig. 14 and Supplementary Table 1). A linear correlation was obtained between $\ln \left(R_{\mathrm{ct}}{ }^{-1}\right)$ and $T^{-1}$ (Fig. 5b), suggesting the applicability of Arrhenius equation of $\ln \left(R_{\mathrm{ct}}{ }^{-1}\right)=-E_{\mathrm{a}} / R T+C^{58,59}$. The activation energy $E_{\mathrm{a}}$ for charge transfer processes was calculated to be $288 \mathrm{meV}$. The low value suggests facile ion desolvation thanks to the help of amino groups.

The proton conduction in TABQ was studied by galvanostatic intermittent titration technique (GITT) at various temperatures. The obtained diffusion coefficient $(D)$ values were used to calculate the activation energy for ion diffusion according to the Arrhenius equation of $\ln (D)=-E_{\mathrm{a}}{ }^{\prime} / R T+C^{\prime}$ (Fig. $5 \mathrm{c}$ and Supplementary Fig. 15$)^{60}$. It results in a low $E_{\mathrm{a}}{ }^{\prime}$ value of $181 \mathrm{meV}$. Considering the hydrogen bonding network formed among TABQ molecules as discussed earlier, the result demonstrates a unique proton conduction manner via the Grotthuss-type mechanism $(E<400 \mathrm{meV})^{61,62}$, where proton transfer takes place by forming/breaking of hydrogen bonds between adjacent carbonyl/hydroxyl and amino groups (Fig. 5d). Such process allows much more facile ion conduction in comparison to the conventional diffusion mode. The charge/discharge kinetics of TABQ were studied by the method proposed by Dunn et al. ${ }^{63}$. The relationship of the peak current $i$ and scan rate $v$ from cyclic voltammetry (CV) obeys the equation of $i=a v^{b}$. The $b$ values of the two pairs of TABQ redox peaks are close to 1 (Supplementary Fig. 16), suggesting the domination of non-diffusion-controlled process and indicating facile reaction kinetics ${ }^{62,63}$. It guarantees the excellent electrochemical performance of the $\mathrm{Zn}$-TABQ battery.

\section{Discussion}

In this work, specific functional groups are chosen for the small molecule quinone cathode of TABQ for aqueous zinc-organic batteries. The hydrogen bonds suppress the sublimation and provides convenience for various electrode treatments. The high molecular symmetry results in low solubility in aqueous electrolytes. More importantly, the redox of carbonyl group is associated with dominated proton de/insertion thanks to the amino groups which undergoes protonation in the weakly acidic zinc electrolyte. Facile proton conduction in TABQ is furthermore achieved with a Grotthuss-type mechanism through the hydrogen bonding network formed with the amino and carbonyl groups. Therefore, proton insertion in TABQ experiences much enhanced kinetics and is favored for energy storage. Despite the low concentration of proton in the electrolyte, the continuous hydrolysis of $\mathrm{Zn}^{2+}$ generates sufficient proton for insertion. The unique proton conduction manner results in activation energies below $300 \mathrm{meV}$ for charge transfer and proton diffusion, which guarantees a high capacity of $303 \mathrm{mAh} \mathrm{g}^{-1}$ with excellent rate capability for the TABQ electrode. Stable cycling is also achieved for 1000 cycles. The unique proton conduction manner presented in our work 


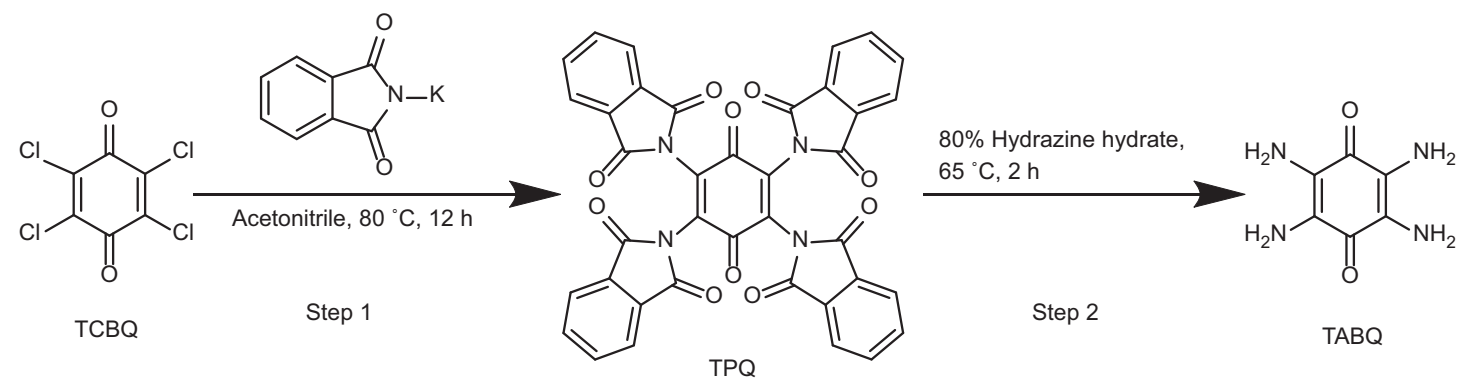

Fig. 6 Synthetic routes of TABQ. TABQ was obtained by a two-step synthesis with low-cost reactants.

proposes a promising direction for the design of highperformance organic electrode materials for aqueous batteries.

\section{Methods}

Materials. Graphite foil was purchased from the SGL group (Germany). TCBQ, THBQ, o-TCBQ, DABQ, DOBDA, benzoquinone and potassium phthalimide were purchased from Aladdin Bio-Chem Technology (China). KB conductive additive was purchased from Lion Specialty Chemicals (Japan). Poly(vinylidene fluoride) (PVDF) was purchased from Kejing Materials Technology (China). The other reagents were obtained from Sinopharm Chemical Reagent (China).

Synthesis of TABQ. The TABQ was prepared according to a previously reported method $^{64}$ as summarized in Fig. 6 and described below.

Step 1: $20.0 \mathrm{~g}(0.081 \mathrm{~mol})$ of TCBQ, $60.0 \mathrm{~g}(0.32 \mathrm{~mol})$ of potassium phthalimide and $200.0 \mathrm{~mL}$ of acetonitrile were added into a $500 \mathrm{~mL}$ round bottom flask. The mixture was kept at $80^{\circ} \mathrm{C}$ for $12 \mathrm{~h}$ under stirring. After cooling down to room temperature, the solid was separated by filtration and washed with $2 \mathrm{~L}$ boiled water. The product was dried at $60^{\circ} \mathrm{C}$ overnight, and brown-yellow powder of tetra (phthalimido)-benzoquinone (TPQ) was obtained $(45.0 \mathrm{~g}$, yield $=80.7 \%)$.

Step 2: $9.41 \mathrm{~g}(0.014 \mathrm{~mol})$ of TPQ was dispersed in $200 \mathrm{~mL}$ of $80 \%$ hydrazine hydrate in a $500 \mathrm{~mL}$ round bottom flask. The mixture was stirred at room temperature for $2 \mathrm{~h}$, followed by heating at $65^{\circ} \mathrm{C}$ for $2 \mathrm{~h}$. The system was cooled down to room temperature, filtered, washed with water and ethanol, and the dark purple product of TABQ was obtained $(1.3 \mathrm{~g}$, yield $=56.6 \%) .{ }^{1} \mathrm{H}$ NMR, $\left(500 \mathrm{MHz},\left[\mathrm{D}_{6}\right]\right.$ DMSO): $\delta 4.55$ (s, 8H) (Supplementary Fig. 17); elemental analysis (calcd., found for $\left.\mathrm{C}_{6} \mathrm{H}_{8} \mathrm{~N}_{4} \mathrm{O}_{2}\right)$ : C (42.86, 42.09), H (4.80, 4.03), N (33.32, 33.27), O (19.03, 21.41).

Characterizations. FT-IR was performed on VERTEX70 (Bruker, Germany). XRD was carried out on an Empyrean diffractometer with $\mathrm{Cu}-\mathrm{Ka}$ radiation (PANalytical B.V., Holland). ${ }^{1} \mathrm{H}$ NMR was measured on Bruker $500 \mathrm{M}$ (Bruker, Germany) with (methyl sulfoxide) $-\mathrm{d}_{6}$ as the solvent and tetramethylsilane (TMS) as the internal standard. Elemental analysis was determined by an Elementar Vario EL III (Elementar, Germany). TGA was carried out on a TGA/DSC3+ thermal analysis system (Mettler toledo, Switzerland). The morphology was obtained by a SU8010 SEM equipped with an EDS detector (HITACHI, Japan). UV-vis absorption spectra were recorded on a U-3900 spectrophotometer (HITACHI, Japan). XPS was carried out on a K-Alpha + X-ray Photoelectron Spectroscopy (Thermo fisher Scientific, America). ICP-OES analysis was carried out on ICP-OES 730 (Agilent, America). IC analysis was carried out on ICS-1100 (DIONEX, America).

Sublimation behavior study. The benzoquinone or TABQ powder was placed on the aluminum foil cover of a long-neck round-bottom flask. It was placed upside down with the powder and neck sticking into the oven which was set at $90^{\circ} \mathrm{C}$ or $150^{\circ} \mathrm{C}$ and the round part staying outside at room temperature. Sublimation was evidenced by the disappearance of powder on the aluminum cover and condensation of crystals on the round part.

UV-vis analysis. Standard solutions were prepared by dissolving known compound concentrations in $1 \mathrm{M} \mathrm{ZnSO}_{4}$. Saturated solutions were obtained by mixing an excess of compounds in $1 \mathrm{M} \mathrm{ZnSO}_{4}$ and filtered. Saturated solutions were diluted by the factors of 100,200 and 100 with $1 \mathrm{M} \mathrm{ZnSO}_{4}$ for TABQ, THBQ and o-TCBQ, respectively. UV-vis absorbance was measured in the range of 190-600 $\mathrm{nm}$. Calibration curves were obtained by the linear fit of the absorbance at $365 \mathrm{~nm}$, $363 \mathrm{~nm}$ and $335 \mathrm{~nm}$ for TABQ, THBQ and o-TCBQ, respectively, with respect to the compound concentrations of standard solutions using the Beer-Lambert law: $A$ $=\varepsilon l c(A$ : absorbance; $\varepsilon$ : molar extinction coefficient; $l$ : length of the cell; $c$ : compound concentration).

Electrochemical measurements. The TABQ electrodes were prepared by mixing TABQ, KB conductive additive, and PVDF binder with a weight ratio of 5:4:1 in NMP. The slurry was drop casted on graphite foil discs with the diameter of 11 mm, and dried at $60^{\circ} \mathrm{C}$ under vacuum overnight followed by $90^{\circ} \mathrm{C}$ overnight. The mass loading of TABQ was around $1.3 \mathrm{mg} \mathrm{cm}^{-2}$. Galvanostatic charge/discharge measurements were carried out in PFA Swagelok ${ }^{\circledR}$ type cells with titanium rods as the current collectors. Rest test was performed with the following process: regular discharge and charge for 20 cycles at $1 \mathrm{~A} \mathrm{~g}^{-1}$, rest for $10 \mathrm{~h}$ after discharged to half capacity, fully discharged, charged to half capacity and fully charged, respectively (a total of $40 \mathrm{~h}$ rest period), and return to regular cycles. The study of electrolyte $\mathrm{pH}$ influence was carried out in T-shaped PFA Swagelok ${ }^{\otimes}$ type cells with TABQ working electrode, graphite foil counter electrode, SCE reference electrode and the aqueous electrolytes of $1 \mathrm{M} \mathrm{ZnSO}_{4}, 1 \mathrm{M} \mathrm{Zn}\left(\mathrm{CF}_{3} \mathrm{SO}_{3}\right)_{2}, 5 \times 10^{-5} \mathrm{M} \mathrm{H}_{2} \mathrm{SO}_{4}, 0.5 \mathrm{M}$ $\mathrm{H}_{2} \mathrm{SO}_{4}$, or $1 \mathrm{M} \mathrm{ZnSO}_{4}+0.5 \mathrm{M} \mathrm{H}_{2} \mathrm{SO}_{4}$. EIS measurements were carried out in a Tshaped PFA Swagelok type cell with TABQ working electrode, $\mathrm{Zn}$ counter electrode, SCE reference electrode and $1 \mathrm{M} \mathrm{ZnSO}_{4}$ aqueous electrolyte. GITT was carried out in a T-shaped PFA Swagelok ${ }^{\circ}$ type cell with TABQ working electrode, $\mathrm{Zn}$ counter electrode, $\mathrm{Zn}$ reference electrode and $1 \mathrm{M} \mathrm{ZnSO}_{4}$ aqueous electrolyte. The cells for EIS and GITT measurements were placed in an oven which was set to the desired temperatures of $35,40,45$, and $50^{\circ} \mathrm{C}$. All electrochemical experiments were carried out on a Bio-Logic VMP3.

In-situ XRD analysis. The test was carried out with a home-made in-situ XRD cell. The TABQ slurry was drop casted on a carbon cloth substrate. The cell was discharged and charged to the desired voltages and XRD measurements were taken

Activation energy calculation. The Nyquist plots from EIS were fitted with a typical equivalent circuit shown in Supplementary Fig. 14b, and the obtained $R_{\mathrm{ct}}$ values were summarized in Supplementary Table 1. The low errors confirmed the applicability of the circuit for calculating $R_{\mathrm{ct}}$. The $\ln \left(R_{\mathrm{ct}}{ }^{-1}\right)$ values were plotted vs. $1000 / T$ and linear fit was carried out according to the Arrhenius equation of $\ln$ $\left(R_{\mathrm{ct}}{ }^{-1}\right)=-E_{\mathrm{a}} / R T+C$, where $C$ is constant under a stable experimental condition, $R$ is gas constant and $T$ is temperature ${ }^{62}$. The $E_{\mathrm{a}}$ represents the activation energy for charge transfer and was calculated from the slope of the fitted line. Similarly, the activation energy $E_{\mathrm{a}}$ ' for diffusion was calculated from the Arrhenius equation with diffusion coefficient $(D)$ of $\ln (D)=-E_{\mathrm{a}}{ }^{\prime} / R T+C^{\prime}$. $D$ was calculated from GITT based on the following equation:

$$
D=\frac{4 L^{2}}{\pi \tau}\left(\frac{\Delta E_{s}}{\Delta E_{\mathrm{t}}}\right)^{2}
$$

where $\tau$ is the relaxation time, $\Delta E_{\mathrm{s}}$ is the steady-state potential change after a single pulse, and $\Delta E_{\mathrm{t}}$ is the potential change during a pulse after eliminating iR drop. The diffusion length $L$ was measured by the geometric thickness of cathode. Since $L$ was a constant, the value would not affect the activation energy obtained from slope of the Arrhenius equation. The linearity between cell voltage and $t^{1 / 2}$ during titration was checked to confirm the applicability of the equation (Supplementary Fig. 18) ${ }^{65}$

Kinetics studies. The charge/discharge kinetics of TABQ in aqueous zinc cells were studied by CV tests at various scan rates (Supplementary Fig. 16a). The CV curves showed two pairs of redox peaks. The peak current $i$ and scan rate $v$ obeys the relationship of $i=a v^{b}$, where $a$ and $b$ are coefficients. In the limiting cases, a $b$ value of 0.5 suggests diffusion-controlled process whereas 1 indicates nondiffusion-controlled process ${ }^{62,63}$. The peak current at different scan rates was extracted, and the ln of peak current was plotted vs. In of scan rate. By carrying out linear fit, the $b$ values were obtained from the slopes of the lines (Supplementary Fig. 16b).

\section{Data availability}

The data that support the findings of this study are available from the corresponding author upon reasonable request.

Received: 8 January 2021; Accepted: 5 July 2021; Published online: 20 July 2021 


\section{References}

1. Armand, M. \& Tarascon, J. M. Building better batteries. Nature 451, 652-657 (2008).

2. Goodenough, J. B. \& Kim, Y. Challenges for rechargeable Li batteries. Chem. Mater. 22, 587-603 (2010).

3. Grey, C. P. \& Hall, D. S. Prospects for lithium-ion batteries and beyond - a 2030 vision. Nat. Commun. 11, 6279 (2020).

4. Xie, J. \& Lu, Y. C. A retrospective on lithium-ion batteries. Nat. Commun. 11, 2499 (2020).

5. Ji, X. L. A paradigm of storage batteries. Energy Environ. Sci. 12, 3203-3224 (2019).

6. Cheng, X. B., Zhang, R., Zhao, C. Z. \& Zhang, Q. Toward safe lithium metal anode in rechargeable batteries: a review. Chem. Rev. 117, 10403-10473 (2017).

7. Nayak, P. K., Yang, L. T., Brehm, W. \& Adelhelm, P. From lithium-ion to sodium-ion batteries: advantages, challenges, and surprises. Angew. Chem. Int. Ed. 57, 102-120 (2018).

8. Zhao, J. et al. High-performance flexible quasi-solid-state zinc-ion batteries with layer-expanded vanadium oxide cathode and zinc/stainless steel mesh composite anode. Nano Energy 62, 94-102 (2019).

9. Lu, Y. \& Chen, J. Prospects of organic electrode materials for practical lithium batteries. Nat. Rev. Chem. 4, 127-142 (2020).

10. Hou, J. et al. Thermal runaway of Lithium-ion batteries employing LiN $\left(\mathrm{SO}_{2} \mathrm{~F}\right)_{2}$-based concentrated electrolytes. Nat. Commun. 11, 5100 (2020).

11. Tang, B. Y., Shan, L. T., Liang, S. Q. \& Zhou, J. Issues and opportunities facing aqueous zinc-ion batteries. Energy Environ. Sci. 12, 3288-3304 (2019).

12. Nam, K. W. et al. Conductive 2D metal-organic framework for highperformance cathodes in aqueous rechargeable zinc batteries. Nat. Commun. 10, 4948 (2019).

13. Blanc, L. E., Kundu, D. \& Nazar, L. F. Scientific challenges for the implementation of Zn-ion batteries. Joule 4, 771-799 (2020).

14. Fang, G. Z., Zhou, J., Pan, A. Q. \& Liang, S. Q. Recent advances in aqueous zinc-ion batteries. ACS Energy Lett. 3, 2480-2501 (2018).

15. Zhang, N. et al. Rechargeable aqueous zinc-manganese dioxide batteries with high energy and power densities. Nat. Commun. 8, 405 (2017).

16. Wan, F. \& Niu, Z. Q. Design strategies for vanadium-based aqueous zinc-ion batteries. Angew. Chem. Int. Ed. 58, 16358-16367 (2019).

17. Zhang, Y. et al. A chemically self-charging aqueous zinc-ion battery. Nat. Commun. 11, 2199 (2020)

18. Xu, C. J., Li, B. H., Du, H. D. \& Kang, F. Y. Energetic zinc ion chemistry: the rechargeable zinc ion battery. Angew. Chem. Int. Ed. 51, 933-935 (2012).

19. Pan, H. L. et al. Reversible aqueous zinc/manganese oxide energy storage from conversion reactions. Nat. Energy 1, 16039 (2016).

20. Kundu, D., Adams, B. D., Duffort, V., Vajargah, S. H. \& Nazar, L. F. A highcapacity and long-life aqueous rechargeable zinc battery using a metal oxide intercalation cathode. Nat. Energy 1, 16119 (2016).

21. Xia, C., Guo, J., Li, P., Zhang, X. X. \& Alshareef, H. N. Highly stable aqueous zinc-ion storage using a layered calcium vanadium oxide bronze cathode. Angew. Chem. Int. Ed. 57, 3943-3948 (2018).

22. Cui, J. et al. Organic cathode materials for rechargeable zinc batteries: mechanisms, challenges, and perspectives. Chemsuschem 13, 2160-2185 (2020).

23. Tie, Z. W. \& Niu, Z. Q. Design strategies for high-performance aqueous $\mathrm{Zn} /$ organic batteries. Angew. Chem. Int. Ed. 59, 21293-21303 (2020).

24. Lu, Y., Zhang, Q., Li, L., Niu, Z. Q. \& Chen, J. Design strategies toward enhancing the performance of organic electrode materials in metal-ion batteries. Chem 4, 2786-2813 (2018).

25. Esser, B. et al. A perspective on organic electrode materials and technologies for next generation batteries. J. Power Sources 482, 228814 (2021).

26. Koshika, K., Sano, N., Oyaizu, K. \& Nishide, H. An aqueous, electrolyte-type, rechargeable device utilizing a hydrophilic radical polymer-cathode. Macromol. Chem. Phys. 210, 1989-1995 (2009).

27. Shi, H.-Y., Ye, Y. J., Liu, K., Song, Y. \& Sun, X. Q. A long-cycle-life self-doped polyaniline cathode for rechargeable aqueous zinc batteries. Angew. Chem. Int. Ed. 57, 16359-16363 (2018).

28. Zhao, Q. et al. High-capacity aqueous zinc batteries using sustainable quinone electrodes. Sci. Adv. 4, eaao1761 (2018).

29. Nam, K. W. et al. Redox-active phenanthrenequinone triangles in aqueous rechargeable zinc batteries. J. Am. Chem. Soc. 142, 2541-2548 (2020).

30. Guo, Z. W. et al. An environmentally friendly and flexible aqueous zinc battery using an organic cathode. Angew. Chem. Int. Ed. 57, 11737-11741 (2018).

31. Park, M. J., Asl, H. Y. \& Manthiram, A. Multivalent-ion versus proton insertion into battery electrodes. ACS Energy Lett. 5, 2367-2375 (2020)

32. Tie, Z. W., Liu, L. J., Deng, S. Z., Zhao, D. B. \& Niu, Z. Q. Proton insertion chemistry of a zinc-organic battery. Angew. Chem. Int. Ed. 59, 4920-4924 (2020).
33. Tie, Z. W. \& Niu, Z. Q. Design strategies for high-performance aqueous Zn/ organic. batteries. Angew. Chem. Int. Ed. 59, 21293-21303 (2020).

34. Alt, H., Binder, H., Köhling, A. \& Sandstede, G. Investigation into the use of quinone compounds-for battery cathodes. Electrochim. Acta 17, 873-887 (1972).

35. Zhao, E. W. et al. In situ NMR metrology reveals reaction mechanisms in redox flow batteries. Nature 579, 224-228 (2020).

36. Liang, $Y$. et al. Universal quinone electrodes for long cycle life aqueous rechargeable batteries. Nat. Mater. 16, 841-848 (2017).

37. Sieuw, L. et al. A H-bond stabilized quinone electrode material for $\mathrm{Li}$-organic batteries: the strength of weak bonds. Chem. Sci. 10, 418-426 (2019).

38. Tomida, S. et al. Infrared spectroscopic analysis on structural changes around the protonated Schiff base upon retinal isomerization in light-driven sodium pump KR2. BBA-Bioenerg. 1861, 148190 (2020).

39. Ledeti, I. et al. Thermal stability of synthetic thyroid hormone L-thyroxine and L-thyroxine sodium salt hydrate both pure and in pharmaceutical formulations. J. Pharm. Biomed. 125, 33-40 (2016).

40. Giubertoni, G., Sofronov, O. O. \& Bakker, H. J. Effect of intramolecular hydrogen-bond formation on the molecular conformation of amino acids. Commun. Chem. 3, 84 (2020).

41. Kundu, D. et al. Organic cathode for aqueous Zn-ion batteries: taming a unique phase evolution toward stable electrochemical cycling. Chem. Mater. 30, 3874-3881 (2018).

42. Khayum, M. A. et al. Zinc ion interactions in a two-dimensional covalent organic framework based aqueous zinc ion battery. Chem. Sci. 10, 8889-8894 (2019).

43. Dawut, G., Lu, Y., Miao, L. C. \& Chen, J. High-performance rechargeable aqueous $\mathrm{Zn}$-ion batteries with a poly(benzoquinonyl sulfide) cathode. Inorg. Chem. Front. 5, 1391-1396 (2018)

44. Wang, Y. R. et al. Binding zinc ions by carboxyl groups from adjacent molecules toward long-life aqueous zinc-organic batteries. Adv. Mater. 32 2000338 (2020).

45. Wang, J. et al. Conjugated sulfonamides as a class of organic lithium-ion positive electrodes. Nat. Mater. 20, 665-673 (2021).

46. Hou, Q., Zhu, L. Q., Chen, H. N., Liu, H. C. \& Li, W. P. Growth of porous $\mathrm{ZnO}$ nanosheets by electrodeposition with $\mathrm{Zn}_{4} \mathrm{SO}_{4}(\mathrm{OH})_{6} \cdot 4 \mathrm{H}_{2} \mathrm{O}$ as precursor. Electrochim. Acta 85, 438-443 (2012).

47. Lee, B. et al. Critical role of $\mathrm{pH}$ evolution of electrolyte in the reaction mechanism for rechargeable zinc batteries. ChemSusChem 9, 2948-2956 (2016).

48. Wan, F. et al. Aqueous rechargeable zinc/sodium vanadate batteries with enhanced performance from simultaneous insertion of dual carriers. Nat. Commun. 9, 1656 (2018).

49. Huang, J. et al. Polyaniline-intercalated manganese dioxide nanolayers as a high-performance cathode material for an aqueous zinc-ion battery. Nat. Commun. 9, 2906 (2018).

50. Yang, J. S., Chiou, S. Y. \& Liau, K. L. Fluorescence enhancement of trans-4 aminostilbene by N-phenyl substitutions: The "amino conjugation effect". $J$. Am. Chem. Soc. 124, 2518-2527 (2002).

51. Janic, I. \& Kakas, M. Electronic configuration and spectra of the neutral and protonated forms of triphenylamine. J. Mol. Struct. 114, 249-252 (1984)

52. Zhang, Q. E., Zhou, A. A., Wang, J. J., Wu, J. F. \& Bai, H. Degradationinduced capacitance: a new insight into the superior capacitive performance of polyaniline/graphene composites. Energ. Environ. Sci. 10, 2372-2382 (2017).

53. Sieuw, L. et al. Through-space charge modulation overriding substituent effect: rise of the redox potential at $3.35 \mathrm{~V}$ in a lithium-phenolate stereoelectronic isomer. Chem. Mater. 32, 9996-10006 (2020).

54. Jin, S. J. et al. A water-miscible quinone flow battery with high volumetric capacity and energy density. ACS Energy Lett. 4, 1342-1348 (2019).

55. Laviron, E. Electrochemical reactions with protonations at equilibrium: Part $\mathrm{X}$. The kinetics of the $\mathrm{p}$-benzoquinone/hydroquinone couple on a platinum electrode. J. Electroanal. Chem. 164, 213-227 (1984).

56. Takashi, H. On the color-reactions of the condensed polycyclic aromatic hydrocarbons and their related quinones in the solutions of the concentrated sulfuric acid. Bull. Chem. Soc. Jpn. 28, 483-489 (1955).

57. Mastragostino, M., Nadjo, L. \& Saveant, J. M. Disproportionation and ECE mechanisms-I. Theoretical analysis. Relationships for linear sweep voltammetry. Electrochim. Acta 13, 721-749 (1968).

58. Mizuno, Y. et al. Suppressed activation energy for interfacial charge transfer of a prussian blue analog thin film electrode with hydrated Ions $\left(\mathrm{Li}^{+}, \mathrm{Na}^{+}\right.$, and $\mathrm{Mg}^{2+}$ ). J. Phys. Chem. C. 117, 10877-10882 (2013).

59. Kundu, D. et al. Aqueous vs. nonaqueous $\mathrm{Zn}$-ion batteries: consequences of the desolvation penalty at the interface. Energy Environ. Sci. 11, 881-892 (2018).

60. Talaie, E. et al. Methods and protocols for electrochemical energy storage materials research. Chem. Mater. 29, 90-105 (2017).

61. Grotthuss, C. J. T. Sur la decomposition de l'eau et des corps q'uelle tient en dissolution à l'aide de l'électricité galvanique. Ann. Chim. LVIII, 54-74 (1806) 
62. Wu, X. Y. et al. Diffusion-free Grotthuss topochemistry for high-rate and long-life proton batteries. Nat. Energy 4, 123-130 (2019).

63. Wang, J., Polleux, J., Lim, J. \& Dunn, B. Pseudocapacitive contributions to electrochemical energy storage in $\mathrm{TiO}_{2}$ (anatase) nanoparticles. J. Phys. Chem. C. 111, 14925-14931 (2007).

64. Luo, Z. Q. et al. A microporous covalent-organic framework with abundant accessible carbonyl groups for lithium-ion batteries. Angew. Chem. Int. Ed. 57, 9443-9446 (2018).

65. Weppner, W. \& Huggins, R. A. Determination of the kinetic parameters of mixed-conducting electrodes and application to the system $\mathrm{Li}_{8} \mathrm{Sb}$. $J$ Electrochem. Soc. 124, 1569-1578 (1977).

\section{Acknowledgements}

This work was supported by the National Natural Science Foundation of China (51974070), the LiaoNing Revitalization Talents Program (XLYC1907069), and the Fundamental Research Funds for the Central Universities (N2105001). Special thanks are due to the instrumental analysis from Analytical and Testing Center, Northeastern University and SDBSWeb: https://sdbs.db.aist.go.jp (National Institute of Advanced Industrial Science and Technology, 2020-09-01).

\section{Author contributions}

Z.L. and X.S. conceived and designed this work. The experiment was performed by Z.L. under the guidance of X.S. and H.-Y.S. with the help of L.L. as well as X.Y.. W.W. carried out the SEM and XRD tests. All co-authors discussed the results. Z.L., X.S. and L.L. wrote the manuscript. All authors have given approval to the final version of the manuscript.

\section{Competing interests}

The authors declare no competing interests.

\section{Additional information}

Supplementary information The online version contains supplementary material available at https://doi.org/10.1038/s41467-021-24701-9.

Correspondence and requests for materials should be addressed to X.S.

Peer review information Nature Communications thanks the anonymous reviewer(s) for their contribution to the peer review of this work. Peer reviewer reports are available.

Reprints and permission information is available at http://www.nature.com/reprints

Publisher's note Springer Nature remains neutral with regard to jurisdictional claims in published maps and institutional affiliations.

(c) Open Access This article is licensed under a Creative Commons Attribution 4.0 International License, which permits use, sharing, adaptation, distribution and reproduction in any medium or format, as long as you give appropriate credit to the original author(s) and the source, provide a link to the Creative Commons license, and indicate if changes were made. The images or other third party material in this article are included in the article's Creative Commons license, unless indicated otherwise in a credit line to the material. If material is not included in the article's Creative Commons license and your intended use is not permitted by statutory regulation or exceeds the permitted use, you will need to obtain permission directly from the copyright holder. To view a copy of this license, visit http://creativecommons.org/ licenses/by/4.0/.

(C) The Author(s) 2021 